\title{
Interval-Valued Intuitionistic Fuzzy Decision-Making Method using Index Matrices and Application in Outsourcing
}

\author{
Velichka Traneva \\ Prof. Asen Zlatarov University \\ 1 Prof. Yakimov Blvd, Burgas 8000, Bulgaria \\ Email: veleka13@gmail.com
}

\author{
Stoyan Tranev \\ Prof. Asen Zlatarov University \\ 1 Prof. Yakimov Blvd, Burgas 8000, Bulgaria \\ Email: tranev@abv.bg
}

\author{
Deyan Mavrov \\ Prof. Asen Zlatarov University \\ 1 Prof. Yakimov Blvd, Burgas 8000, Bulgaria \\ Email: dg@mavrov.eu
}

\begin{abstract}
Selecting a suitable outsourcing service provider is a challenging problem that requires discussion among a group of experts. The problems of this type belongs to the area of multicriteria decision-making. Interval-valued intuitionistic fuzzy sets, which are an extension of intuitionistic fuzzy sets, are a capable tool in modeling uncertain problems. In this paper we will formulate an optimal interval-valued intuitionistic fuzzy multicriteria decision-making problem in outsourcing and propose a new approach for the selection of the most appropriate candidates; as well as a software program for its automated solution, based on our previous libraries. As an example of a case study, an application of the algorithm on real data from a refinery is demonstrated.
\end{abstract}

\section{INTRODUCTION}

T HE AIM of multi-criteria decision-making (MCDM) is to determine an optimal alternative having the highest degree of desirability with respect to all relevant goals [3]. Most decisions are not made on the basis of exact data. Zadeh's Fuzzy Logic [15] has emerged to help model this vague environment. The uncertainty in the MCDM-problem may be caused by unavailable or indeterminate characteristics of the alternative options or from the inability of the experts to formulate a precise evaluation [16]. Atanassov, in 1983, introduced the notion of an intuitionistic fuzzy set (IFS, [5]) as a generalization of fuzzy sets, which adds a degree of hesitance. Later, Atanassov and Gargov proposed in 1989 the concept of interval-valued intuitionistic fuzzy sets (IVIFSs, [7]). There are many papers for application of IVIF theory in MCDMproblems.

In this study, an optimal generalized MCDM-approach (IVIFIMOA) for selecting the most appropriate outsourcing providers will be formulated over IVIF data. In [19], [20] we have proposed an IF algorithm for the selection of outsourcing service providers using the concepts of IMs [4] and

Work is supported by the Asen Zlatarov University through project Ref. No. NIX-440/2020 "Index matrices as a tool for knowledge extraction".
IF logic [5]. Here we will extend this approach to intervalvalued IF (IVIF) logic [7].

The rest of the paper contains the following sections: Section 2 presents IVIFSs and IMs. Section 3 formulates an optimal IVIF problem for the selection of outsourcing providers, gives an algorithm for its solution and describes the software implementation. A real life case study is described. Section 4 concludes the work and gives future suggestions.

\section{BASIC CONCEPTS OF IMS AND IVIF LOGIC}

This section recalls some basic concepts on interval-valued intuitionistic fuzzy pairs (IVIFPs) from [6], [12] and on the index matrix apparatus from [4], [8], [23].

\section{A. Interval-Valued Intuitionistic Fuzzy Logic}

The concept of IVIFPs was introduced in [12]. The IVIFP is an object of the form $\langle M, N\rangle$, where $M, N \subseteq[0,1]$ are closed sets, $M=[\inf M, \sup M], N=[\inf N, \sup N]$ and

$$
\sup M+\sup N \leq 1
$$

that is used as an evaluation of some object or process and whose components ( $M$ and $N$ ) are interpreted as intervals of degrees of membership and non-membership, or intervals of degrees of validity and non-validity, etc.

Let us have two IVIFPs $x=\langle M, N\rangle$ and $y=\langle P, Q\rangle$. In [6], [12] are defined the operations classical negation, conjunction, disjunction, multiplication with constant, and difference.

The forms of the relations with IVIFPs are given in [6].

\section{B. Interval-Valued Intuitionistic Fuzzy Index Matrices}

Let $\mathscr{I}$ be a fixed set. Three-dimensional interval-valued intuitionistic fuzzy index matrix (3-D IVIFIM) with index sets $K, L$ and $H(K, L, H \subset \mathscr{I})$, we denote the object [4], [8]:

$$
\left[K, L, H,\left\{\left\langle M_{k_{i}, l_{j}, h_{g}}, N_{k_{i}, l_{j}, h_{g}}\right\rangle\right\}\right]
$$




\begin{tabular}{c|ccc}
$h_{g} \in H$ & $l_{1}$ & $\ldots$ & $l_{n}$ \\
\hline$k_{1}$ & $\left\langle M_{k_{1}, l_{1}, h_{g}}, M_{k_{1}, l_{1}, h_{g}}\right\rangle$ & $\ldots$ & $\left\langle M_{k_{1}, l_{n}, h_{g}}, N_{k_{1}, l_{n}, h_{g}}\right\rangle$ \\
$\vdots$ & $\vdots$ & $\ddots$ & $\vdots$ \\
$k_{m}$ & $\left\langle M_{k_{m}, l_{1}, h_{g}}, N_{k_{m}, l_{1}, h_{g}}\right\rangle$ & $\ldots$ & $\left\langle M_{k_{m}, l_{n}, h_{g}}, N_{k_{m}, l_{n}, h_{g}}\right\rangle$
\end{tabular},

where for every $1 \leq i \leq m, 1 \leq j \leq n, 1 \leq g \leq f$ :

$M_{k_{i}, l_{j}, h_{g}} \subseteq[0,1], N_{k_{i}, l_{j}, h_{g}} \subseteq[0,1], \sup M_{k_{i}, l_{j}, h_{g}}+\sup N_{k_{i}, l_{j}, h_{g}} \leq 1$.

Over every two 3-D IVIFIMs $A$ and $B$ we can apply the operations addition, transposition, multiplication, projection and substitution, as defined in [4], [8], [21]. [22] defines the operation "aggregation by one dimension".

A Level operator for decreasing the number of elements of IVIFM: Let $\langle\alpha, \beta\rangle$ is an IVIFP, then according to [14] $N_{\alpha, \beta}^{>}(A)=\left[K, L, H,\left\{\left\langle R_{k_{i}, l_{j}, h_{g}}, P_{k_{i}, l_{j}, h_{g}}\right\rangle\right\}\right]$, where

$$
\begin{aligned}
& \left\langle R_{k_{i}, l_{j}, h_{g}}, P_{k_{i}, l_{j}, h_{g}}\right\rangle
\end{aligned}
$$

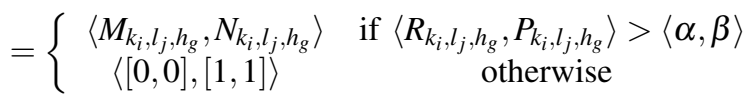

\section{Optimal INTERVAL-VALUED INTUITIONISTIC FUZZY SELECTION FOR THE OUTSOURCING SERVICE PROVIDERS}

Here, we will formulate an optimal IVIF outsourcing problem.

The management team of a company has selected the following activities $v_{e}(1 \leq e \leq u)$ to be offered for outsourcing in order to increase the profitability of the enterprise. An expert team, consisting of experts $\left\{r_{1}, \ldots, r_{s}, \ldots, r_{D}\right\}$ has proposed an evaluation system, giving each candidate $\left\{k_{1}, \ldots, k_{i}, \ldots, k_{m}\right\}$ (for $i=1, \ldots, m$ ) for the respective outsourced service $v_{e}(1 \leq$ $e \leq u)$, an evaluation by each criterion $\left\{c_{1}, \ldots, c_{j}, \ldots, c_{n}\right\}$ (for $j=1, \ldots, n$ ). The weight coefficients of each assessment criteria $c_{j}$ (for $j=1, \ldots, n$ ) according to their priority for the service $v_{e}$ are given in the form of IVIFPs - $p k_{c_{j}, v_{e}}$ (for $j=1, \ldots, n)$. Each expert has an IVIFP rating $r_{s}=\left\langle\Delta_{s}, \varepsilon_{s}\right\rangle$ $(1 \leq s \leq D)$. Let the number of his/her own participations in previous outsourcing procedures be equal to $\Gamma_{s}(s=1, \ldots, D)$, respectively. All applicants need to be evaluated by the team of experts according to the established criteria in the company at the current time point $h_{f}$ for their application for each outsourced service $v_{e}(1 \leq e \leq u)$, and their evaluations $e v_{k_{i}, c_{j}, d_{s}}$ (for $1 \leq i \leq m, 1 \leq j \leq n, 1 \leq s \leq D$ ) are IVIFPs. Now we need to find the optimal assignment of candidates.

\section{A. Optimal IVIF Selection of the Providers}

To solve this problem, we propose a new approach - IVIFIMOA, described with mathematical notation and pseudocode: Step 1. This step creates an expert 3-D evaluation IM EV. It is possible for the experts to include assessments for the same candidates from a previous evaluation IM at time points $h_{1}, \ldots, h_{g}, \ldots, h_{f-1}$. The team of experts needs to evaluate the candidates for the services according to the approved criteria in the company at the current time moment $h_{f}$. The experts are uncertain about their evaluations due to changes in some uncontrollable factors. The evaluations are IVIFPs.

It is possible that some of the experts' assessments are incorrect from an IVIF point of view. In [7], different ways for altering incorrect experts' estimations are discussed. Let us propose that, the estimations of the $D_{s}(1 \leq s \leq D)$ expert are correct and described by the IVIFIM $E \bar{V}_{s}=\left[K, C, H,\left\{e v_{k_{i}, c_{j}, d_{s}, h_{g}}\right\}\right]$ as follows:

$$
\begin{array}{c|ccc}
h_{g} \in H & c_{1} & \ldots & c_{n} \\
\hline k_{1} & \left\langle M_{k_{1}, c_{1}, d_{s}, h_{g}}, N_{k_{1}, c_{1}, d_{s}, h_{g}}\right\rangle & \ldots & \left\langle M_{k_{1}, c_{n}, d_{s}, h_{g}}, N_{\left.k_{1}, c_{n}, d_{s}, h_{g}\right\rangle}\right\rangle \\
\vdots & \left\langle M_{k_{m}, c_{1}, d_{s}, h_{g}} ; N_{k_{m}, c_{1}, d_{s}, h_{g}}\right\rangle & \ldots & \left\langle M_{k_{m}, c_{n}, d_{s}, h_{g} ;} ; N_{k_{m}, c_{n}, d_{s}, h_{g}}\right\rangle
\end{array}
$$

where $\quad K=\left\{k_{1}, k_{2}, \ldots, k_{m}\right\}, \quad C=\left\{c_{1}, c_{2}, \ldots, c_{n}\right\}, H=$ $\left\{h_{1}, h_{2}, \ldots, h_{f}\right\}$ and IVIFP $\left\{e v_{\left.k_{i}, c_{j}, d_{s}, h_{g}\right\}}\right.$ is the estimate of the $d_{s}$-th expert for the $k_{i}$-th candidate by the $c_{j}$-th criterion at a moment $h_{g}$.

Let us apply the $\alpha_{H}$-th aggregation operation $\alpha_{E V_{s}, \#_{q}}$ to find the evaluation of the $d_{s}$-th expert $(s=1, \ldots, D)$, where $1 \leq q \leq$ 3. We get the 3-D IVIFIM $E V\left[K, C, E,\left\{e v_{k_{i}, c_{j}, d_{s}}\right\}\right]$ with the evaluations of all experts for all candidates:

$$
\begin{gathered}
E V=\alpha_{E V_{1}, \#_{q}}\left(H, d_{1}\right) \oplus_{(\max , \min )} \oplus_{(\max , \min )} \cdots \\
\ldots \oplus_{(\max , \min )} \alpha_{E V_{D}, \#_{q}}\left(H, d_{D}\right)
\end{gathered}
$$

Go to Step 2.

Step 2. Let the score (rating) $r_{s}$ of the $d_{s}$-th expert $\left(d_{s} \in E\right)$ be specified by an IVIFP $\left\langle\delta_{s}, \varepsilon_{s}\right\rangle$. $\delta_{s}$ and $\varepsilon_{s}$ are interpreted respectively as his degree of competence and of incompetence. Then we create $E V^{*}\left[K, C, E,\left\{e v^{*} k_{i}, c_{j}, d_{s}\right\}\right]$ :

$$
E V^{*}=r_{1} p r_{K, C, d_{1}} E V \ldots \oplus_{(\max , \min )} r_{D} p r_{K, C, d_{D}} E V ;
$$

$E V:=E V^{*}\left(e v_{k_{i}, l_{j}, d_{s}}=e v_{k_{i}, l_{j}, d_{s}}^{*}, \forall k_{i} \in K, \forall l_{j} \in L, \forall d_{s} \in E\right)$.

Then $\alpha_{E}$-th aggregation operation is applied to find the aggregated assessment $R=\alpha_{E, \#_{q}}\left(E V, h_{f}\right)(1 \leq q \leq 3)$ of the $k_{i^{-}}$ th candidate against the $c_{j}$-th criterion at the moment $h_{f} \notin E$. If $q$ is 2 or 3 , then the evaluation of the candidates is more optimistic as outsorcing service provider. Go to Step 3.

Step 3. Let us define the 3-D IFIM $P K\left[C, V, h_{f},\left\{p k_{c_{j}, v_{e}, h_{f}}\right\}\right]$ of the weight coefficients of the assessment criterion according to its priority to the outsourcing service $v_{e}(1 \leq e \leq u)$, where $C=\left\{c_{1}, \ldots, c_{n}\right\}, V=\left\{v_{1}, \ldots, v_{u}\right\}$ and all elements $p k_{c_{j}, v_{e}, h_{f}}$ are IVIFPs. The transposed IM of $R$ is founded under the form $R^{T}\left[K, C, h_{f}\right]$ and is calculated 3-D IVIFIM

$$
B\left[K, V, h_{f},\left\{b_{k_{i}, v_{e}, h_{f}}\right\}\right]:=R^{T} \odot_{(\circ, *)} P K,
$$

which contains the cumulative estimates of the $k_{i}$-th candidate (for $1 \leq i \leq m$ ) for the $v_{e}$-th outsourcing service. If a candidate $k_{i}(1 \leq i \leq m)$ does not wish to participate in the competition to provide an outsourcing service $v_{e}$, then the element $b_{k_{i}, v_{e}, h_{f}}$ is equal to $\langle[0,0],[1,1]\rangle$. Go to Step 4.

Step 4. The aggregation operation $\alpha_{K, \#_{q}}\left(B, k_{0}\right)$ is applied by the dimension $K$ to find the most suitable candidate for the outsourcing service $v_{e}$, where $k_{0} \notin K, 1 \leq q \leq 3$.

If the company requires a different candidate for each service, then it is necessary to apply the IVIF Hungarian algorithm [18] to the data contained in the IVIFIM $B$ and then the optimal allocation of the candidates will be found. It is possible to reduce the candidates with an overall score lower than the IVIFP $\langle\alpha, \beta\rangle$ applying the level-operator (2) to IVIFIM $B$ before the algorithm is implemented. Go to Step 5 . 
Step 5. At this step of the algorithm, we need to determine whether there are correlations between some of the evaluation criteria [11]. The procedure of IVIF-form of ICrA (IVIFICrA), based on the intercriteria analysisis [10] is discussed in [13].

Let IVIFP $\langle\alpha, \beta\rangle$ be given. The criteria $C_{k}$ and $C_{l}$ are in: strong $(\alpha, \beta)$-positive consonance, if $\inf M_{C_{k}, C_{l}}>\alpha$ and $\sup N_{C_{k}, C_{l}}<\beta$; weak $(\alpha, \beta)$-positive consonance, if $\sup M_{C_{k}, C_{l}}>\alpha$ and $\inf N_{C_{k}, C_{l}}<\beta$; strong $(\alpha, \beta)$-negative consonance, if $\sup M_{C_{k}, C_{l}}<\alpha$ and $\inf N_{C_{k}, C_{l}}>\beta$; weak $(\alpha, \beta)$ negative consonance, if $\inf M_{C_{k}, C_{l}}<\alpha$ and $\sup N_{C_{k}, C_{l}}>\beta$; $(\alpha, \beta)$-dissonance, otherwise.

After application of the IVIFICrA over IFIM $R$ we determine which criteria are in consonance. Then, we can evaluate their complexity and more expensive or slower criteria can be removed from the evaluation system. If $O=\left\{O_{1}, \ldots, O_{V}\right\}$ are the criteria that can be omitted, then we can reduce $R$ by IM-operation $R *=R_{(O, \perp)}$. Go to Step 6 .

Step 6. The last step determinates the new rating coefficients of the experts. Let the expert $d_{s}(s=1, \ldots, D)$ participate in $\Gamma_{s}$ procedures, on the basis of which his score $r_{s}=\left\langle\Delta_{s}, \varepsilon_{s}\right\rangle$ is determined, then after his participation in $\left(\Gamma_{s}+1\right)$-th procedure his score will be determined by [5]:

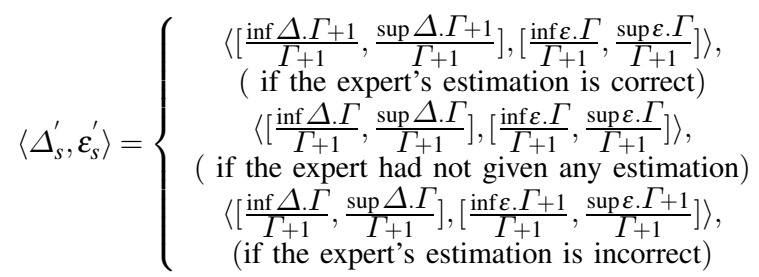

The complexity of the algorithm whithout step 5 is $O(D m n)$ (the complexity of the ICrA in the step 5 is $O\left(m^{2} n^{2}\right)$ [17]).

In order to apply IVIFIMOA algorithm on real data more easily, we are currently developing a command line utility. It is written in $\mathrm{C}++$ and uses an IM template class (IndexMatrix $\langle T\rangle$ ), which implements the basic IM operations [2]. Any type used with the IM class must provide methods for performing operations on the current object and between two objects, so that they can be substituted in the already prepared IM operation methods. As part of a previous work on intuitionistic fuzzy ANOVA [24], we delevoped a class representing IFPs. Using the work done previously, for this project we are developing a class for IVIF pairs.

\section{B. Real life case study}

In this section, the proposed IVIFIMOA approach is applied to a real case study in an oil refinery [1] with the help of the "IVIFIMOA" software utility. The studied refinery adopts the outsourcing model. After the restructuring, the following activities remain outside the company, and will be offered for outsourcing: $v_{1}$ - trade and distribution of high quality fuels, polymers and petrochemicals; $v_{2}$ - engineering activity, specialized in consulting, preparation of technical and economic opinions, detailed projects with author's supervision; $v_{3}$ transport service for public transport of goods and passengers, as well as services with construction machinery; $v_{4}$ - aviation fuel distributor. For this purpose, the refinery invites a team of the experts $d_{1}, d_{2}$ and $d_{3}$ to evaluate the candidates $k_{i}$ (for $1 \leq i \leq 4)$ for the outsourced refinery services. The real evalu- ation system of outsourcing providers selection is determined on the basis of 5 criteria as follows: $C_{1}$ - compliance of the outsourcing service provider with its corporate culture; $C_{2}$ - understanding of the outsourcing service by the provider; $C_{3}$ - necessary resources of the outsourcing provider for the implementation of the service; $C_{4}$ - price of the provided service; $C_{5}$ - opportunity for strategic development of the outsourcing service together with the outsourcing-assignor.

The weight coefficients for the service $v_{e}-p k_{c_{j}, v_{e}}$ for the criteria $c_{j}$ (for $j=1, \ldots, 5$ ) according to their priority for the service $v_{e}(e=1,2,3,4)$ and the ratings of the experts $\left\{r_{1}, r_{2}, r_{3}\right\}$ be given under the form of IVIFPs. The aim of the problem is to optimally select the outsourcing providers.

An optimal solution of the problem:

Step 1. A 3-D expert evaluation IVIFIM $E V\left[K, C, E,\left\{e s_{k_{i}, c_{j}, d_{s}}\right\}\right]$ is created and IVIFP $\left\{e v_{k_{i}, c_{j}, d_{s}}\right\}$ (for $1 \leq i \leq 4,1 \leq j \leq 5,1 \leq s \leq 3$ ) is the estimate of the $d_{s}$-th expert for the $k_{i}$-th candidate by the $c_{j}$-th criterion

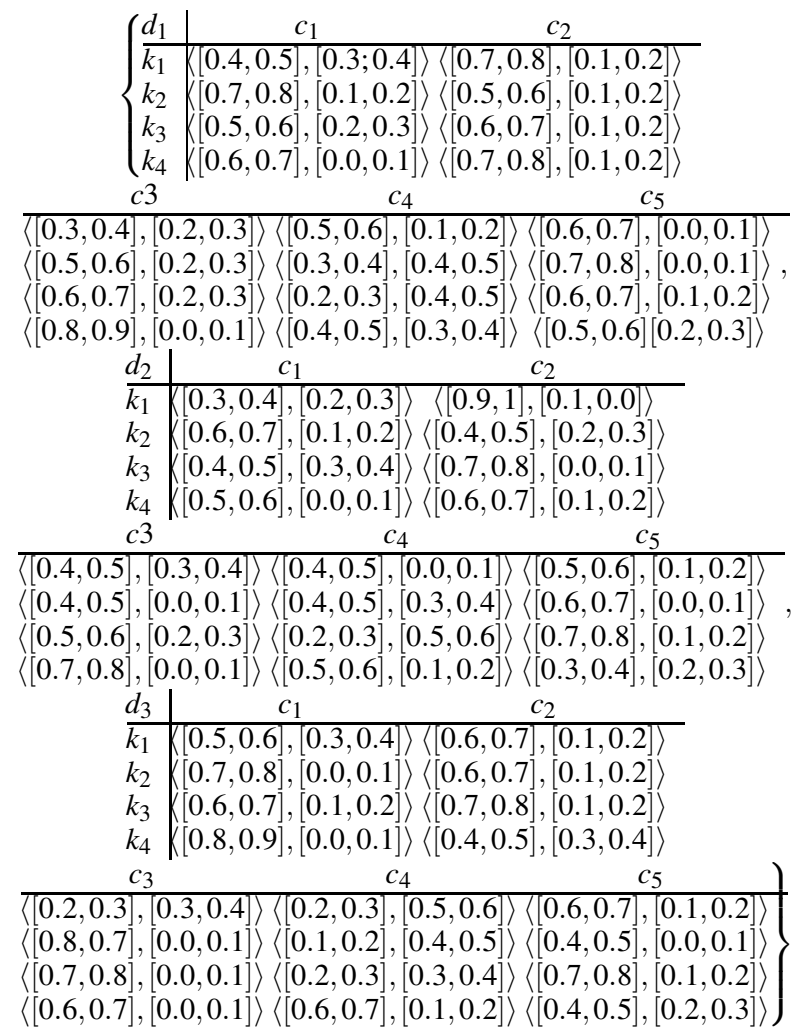

The experts' evaluations are transformed from positive integers to IVIF data using the method described in [7].

Step 2. Let the experts have the following rating coefficients respectively: $\left\{r_{1}, r_{2}, r_{3}\right\}=\{\langle[0.7,0.8],[0.0,0.1]\rangle$,

$\langle[0.6,0.7],[0.0,0.1]\rangle,\langle[0.8,0.9],[0.0,0.1]\rangle\}$.

We create $E V^{*}\left[K, C, E,\left\{e v^{*}\right\}\right]=r_{1} p r_{K, C, d_{1}} E V \oplus_{(\max , \min )}$ $r_{2} p r_{K, C, d_{2}} E V \oplus_{(\max , \min )} r_{3} p r_{K, C, d_{3}} E V$. Then, $E V:=E V^{*}$.

Let us apply the optimistic aggregation operation $\alpha_{E,(\max , \min )}\left(E V, h_{f}\right)=R\left[K, h_{f}, C\right]$ to find the aggregated value of the $k_{i}$-th candidate against the $c_{j}$-th criterion in a current time-moment $h_{f} \notin D$.

Step 3. The 3-D IFIM $P K\left[C, V, h_{f},\left\{p k_{c_{j}, v_{e}, h_{f}}\right\}\right]$ of the weight coefficients of the assessment criterion according to its priority 
to the service $v_{e}(e=1,2,3,4)$ has the following form:

\begin{tabular}{c|cc} 
& $v_{1}$ & $v_{2}$ \\
\hline$c_{1}$ & $\langle[0.8,0.9],[0.0,0.1]\rangle$ & $\langle[0.7,0.8],[0.1,0.2]\rangle$ \\
$c_{2}$ & $\langle[0.7,0.8],[0.0,0.1]\rangle$ & $\langle[0.5,0.6],[0.1,0.2]\rangle$ \\
$c_{3}$ & $\langle[0.5,0.6],[0.1,0.2]\rangle$ & $\langle[0.8,0.9],[0.0,0.1]\rangle$ \\
$c_{4}$ & $\langle[0.8,0.9],[0.0,0.1]\rangle$ & $\langle[0.8,0.9],[0.0,0.1]\rangle$ \\
$c_{5}$ & $\langle[0.7,0.8],[0.1,0.2]\rangle$ & $\langle[0.8,0.9],[0.0,0.1]\rangle$
\end{tabular}

\begin{tabular}{cc}
$v_{3}$ & $v_{4}$ \\
\hline$\langle[0.5,0.6],[0.1,0.2]\rangle$ & $\langle[0.6,0.7],[0.1,0.2]\rangle$ \\
$\langle[0.6,0.7],[0.0,0.2]\rangle$ & $\langle[0.7,0.8],[0.1,0.2]\rangle$ \\
$\langle[0.4,0.5],[0.2,0.3]\rangle$ & $\langle[0.6,0.7],[0.1,0.2]\rangle$ \\
$\langle[0.7,0.8],[0.0,0.1]\rangle$ & $\langle[0.6,0.7],[0.1,0.2]\rangle$ \\
$\langle[0.8,0.9],[0.0,0.1]\rangle$ & $\langle[0.5,0.6],[0.3,0.4]\rangle$
\end{tabular}

where $C=\left\{c_{1}, \ldots, c_{5}\right\}, V=\left\{v_{1}, \ldots, v_{4}\right\}$ and for $1 \leq j \leq 5,1 \leq$ $e \leq 4: p k_{c_{j}, v_{e}, h_{f}}$ are IVIFPs. We construct $B=R^{\bar{T} \odot_{(\circ, *)} P K}$

\begin{tabular}{r|cc} 
& $v_{1}$ & $v_{2}$ \\
\hline$k_{1}$ & $\langle[0.82,0.95],[0,0.003]\rangle$ & $\langle[0.81,0.94],[0,0.004]\rangle$ \\
$k_{2}$ & $\langle[0.87,0.96],[0,0.006]\rangle$ & $\langle[0.89,0.97],[0,0.005]\rangle$ \\
$k_{3}$ & $\langle[0.86,0.97],[0,0.007]\rangle$ & $\langle[0.87,0.97],[0,0.007]\rangle$ \\
$k_{4}$ & $\langle[0.89,0.98],[0,0.006]\rangle$ & $\langle[0.90,0.98],[0,0.006]\rangle$
\end{tabular}

\begin{tabular}{cc}
$v_{3}$ & $v_{4}$ \\
\hline$\langle[0.77,0.92],[0,0.005]\rangle$ & $\langle[0.76,0.91],[0.0004,0.01]\rangle$ \\
$\langle[0.81,0.93],[0,0.009]\rangle$ & $\langle[0.82,0.94],[0.0002,0.02]\rangle$, \\
$\langle[0.81,0.95],[0,0.01]\rangle$ & $\langle[0.81,0.95],[0.0003,0.02]\rangle$ \\
$\langle[0.82,0.95],[0.00004,0.01]\rangle$ & $\langle[0.84,0.96],[0.0002,0.01]\rangle$
\end{tabular}

which contains the cumulative optimistic estimates of the $k_{i}$-th candidate (for $1 \leq i \leq 4$ ) for the $v_{e}$-th vacancy (for $1 \leq e \leq 4$ ). Step 4. We apply the optimistic aggregation operation. We can conclude that $k_{4}$ is the optimal outsourcing provider for all services, respectively: $v_{1}$ - with degree of acceptance (d.a.) $\in[0.89,0.98] ; v_{2}$ - with d.a. $\in[0.9,0.98] ; v_{3}$ - with d.a. $\in[0.82,0.95]$ and $v_{4}$ - with d.a. $\in[0.84,0.96]$.

After application of IVIF Hungarian algorithm [18], we find that $k_{1}$ is the optimal provider for service $v_{3}, k_{2}$ - for the service $v_{1}, k_{3}$ - for the service $v_{4}$ and $k_{4}$ - for the service $v_{2}$. Step 5. After application of the interval-valued form of ICrA with $\alpha=[0.80 ; 0.90]$ and $\beta=[0 ; 0.10]$ over $R$ we determine that there are not criteria in a consonance.

Step 6. If all experts' estimations are correct then we obtain their new ratings as follows: for the expert $d_{1}$ $\left\{\langle[0.73 ; 0.82][0 ; 0.09]\rangle\right.$, for the $d_{2}-\{\langle[0.64 ; 0.73][0 ; 0.09]\rangle$ and for the $d_{3}-\{\langle[0.82 ; 0.91][0 ; 0.09]\rangle$.

\section{CONCLUSION}

We have applied our newly proposed IVIFIMOA algorithm on real data from an oil refinery and have shown how it can be used to select the most eligible candidates for outsourcing company services. The proposed algorithm can be easily generalized for multidimensional IF data [9] and can be applied to MCDM with both exact and IF parameters. For future work we will further develop the software utility and will analyse more real datasets.

\section{REFERENCES}

[1] S. Tranev, Outsourcing conflicts in the transport infrastructure of a company, Dissertation. Prof. Dr. A. Zlatarov University, Burgas; 2012 (in Bulgarian).

[2] D. Mavrov, Software Implementation and Applications of Index Matrices, Dissertation. Prof. Dr. A. Zlatarov University, Burgas; 2016 (in Bulgarian).
[3] H. J. Zimmerman,, Fuzzy sets, decision making, and expert systems, Boston: Kluwer Academic Publishers; 1987.

[4] K. Atanassov, Index Matrices: Towards an Augmented Matrix Calculus. Studies in Computational Intelligence, Springer, Cham, vol. 573; DOI: 10.1007/978-3-319-10945-9, 2014.

[5] K. Atanassov, "On Intuitionistic Fuzzy Sets Theory," STUDFUZZ, vol. 283, Springer, Heidelberg, DOI: 10.1007/978-3-642-29127-2, 2012.

[6] K. Atanassov, "Interval-valued intuitionistic fuzzy sets," Studies in Fuzziness and Soft Computing, Springer, vol. 388, 2020.

[7] K. Atanassov, G. Gargov, "Interval valued intuitionistic fuzzy sets," Fuzzy sets and systems, vol. 31 (3), 1989, pp. 343-349.

[8] K. Atanassov, "Extended Interval Valued Intuitionistic Fuzzy Index Matrices," In: Atanassov K. et al. (eds) Uncertainty and Imprecision in Decision Making and Decision Support: New Challenges, Solutions and Perspectives, IWIFSGN 2018, Advances in Intelligent Systems and Computing, vol. 1081, Springer, Cham, 2020.

[9] K. Atanassov, "n-Dimensional extended index matrices Part 1," Advanced Studies in Contemporary Mathematics, vol. 28 (2), 2018, pp. 245-259.

[10] K. Atanassov, D. Mavrov, V. Atanassova, "Intercriteria decision making: a new approach for multicriteria decision making, based on index matrices and intuitionistic fuzzy sets," Issues in IFSs and Generalized Nets, vol. 11, 2014, pp. 1-8.

[11] K. Atanassov, E. Szmidt, J. Kacprzyk, V. Atanassova, "An approach to a constructive simplication of multiagent multicriteria decision making problems via ICrA," Comptes rendus de lAcademie bulgare des Sciences, vol. 70 (8), 2017, pp. 1147-1156.

[12] K. Atanassov, P. Vassilev, J. Kacprzyk, E. Szmidt, "On interval-valued intuitionistic fuzzy pairs," Journal of Universal Mathematics, vol. 1 (3), 2018, pp. 261-268.

[13] K. Atanassov, P. Marinov, V. Atanassova, "InterCriteria analysis with interval-valued intuitionistic fuzzy evaluations," in: Cuzzocrea A., Greco S., Larsen H., Saccà D., Andreasen T., Christiansen H. (eds) Flexible Query Answering Systems, FQAS 2019, Lecture Notes in Computer Science, Springer, Cham, vol. 11529, 2019, pp. 329-338.

[14] K. Atanassov, P. Vassilev, O. Roeva, "Level Operators over Intuitionistic Fuzzy Index Matrices," Mathematics, vol. 9, 2021, pp. 366.

[15] L. Zadeh, Fuzzy Sets, Information and Control, vol. 8 (3), 338-353; 1965.

[16] R. Yager, "Non-numeric multi-criteria multi-person decision making," International Journal of Group Decision Making and Negotiation, vol. 2, 1993, pp. 81-93.

method for logistics outsourcing provider selection," Knowledge-Based Systems, 2015

[17] V. Atanassova, O. Roeva, "Computational complexity and influence of numerical precision on the results of intercriteria analysis in the decision making process," Notes on Intuitionistic Fuzzy Sets, vol. 24 (3), 2018, pp. 53-63.

[18] V. Traneva, S. Tranev, "An Interval-Valued Intuitionistic Fuzzy Approach to the Assignment Problem," In: Kahraman C. et al. (eds) Intelligent and Fuzzy Techniques in Big Data Analytics and Decision Making, INFUS 2019, Advances in Intelligent Systems and Computing, vol. 1029, Springer, Cham, 2020, pp. 1279-1287.

[19] V. Traneva, S. Tranev, "Intuitionistic Fuzzy Index-Matrix Selection for the Outsourcing Providers at a Refinery," INFUS 2021, Advances in Intelligent Systems and Computing, Springer, Cham, 2021.

[20] V. Traneva, S. Tranev, "Intuitionistic Fuzzy Approach for Outsourcing Provider Selection in a Refinery," In: S. Margenov, I. Lirkov (eds.) Proceedings of LSSC 2021, Sozopol, Bulgaria, Lecture Notes in Computer Science, Springer, Cham, 2021 (in press).

[21] V. Traneva, S. Tranev, V. Atanassova, " Three-Dimensional IntervalValued Intuitionistic Fuzzy Appointment Model”, In: S. Fidanova (eds.) Recent Advances in Computational Optimization, Studies in Computational Intelligence, vol. 838, Springer, Cham, 2020, pp. 181-199.

[22] V. Traneva, S. Tranev, M. Stoenchev, K. Atanassov, "Scaled aggregation operations over two- and three-dimensional index matrices," Soft computing, vol. 22, 2019, pp. 5115-5120.

[23] V. Traneva, S. Tranev, Index Matrices as a Tool for Managerial Decision Making, Publ. House of the USB; 2017 (in Bulgarian).

[24] V. Traneva, D. Mavrov, S. Tranev, "Fuzzy Two-Factor Analysis of COVID-19 Cases in Europe," 2020 IEEE 10th International Conference on Intelligent Systems, IS 2020 - Proceedings, 2020, pp. 533-538. 